\title{
Processo de Acreditação das Escolas Promotoras de Saúde em âmbito mundial: revisão sistemática
}

\author{
Process of Accreditation of Health Promoting Schools worldwide: \\ A Systematic Review
}

\author{
Meirele Rodrigues Inácio da Silva ${ }^{1}$ \\ Alinne Paula de Almeida ${ }^{2}$ \\ Juliana Costa Machado ${ }^{2}$ \\ Luciana Saraiva da Silva ${ }^{2}$ \\ Juliana Aparecida Fialho Cardoso ${ }^{3}$ \\ Glauce Dias da Costa ${ }^{2}$ \\ Rosângela Minardi Mitre Cotta ${ }^{2}$
}

\footnotetext{
${ }^{1}$ Departamento de

Educação Física,

Universidade Federal de Juiz de Fora. Av. Doutor Raimundo Rezende 330, Centro. 35012-140 Governador Valadares MG Brasil.

meireler@yahoo.com.br

${ }^{2}$ Departamento de Nutrição e Saúde, Universidade Federal de Viçosa (UFV).

Viçosa MG Brasil.

${ }^{3}$ Departamento de

Administração e

Contabilidade, UFV. Viçosa

MG Brasil.
}

\begin{abstract}
The scope of this article is to analyze the accreditation criteria of the studies that evaluated actions of health promotion and risk factor prevention of Health Promoting Schools (HPS). A systematic review was conducted based on the recommendations proposed in the "Preferred Reporting Items for Systematic Reviews and $\mathrm{Me}$ ta-Analyses (Prisma)" protocol of articles that assessed HPS in the following databases: SciELO, Lilacs, Medline, PubMed and Portal Capes. From the analysis of the three pillars for accreditation of HPS, three of the studies analyzed did not include all the criteria for certification as HPS on the "Planning Process" and "Health Promotion Activities Developed" pillars. The schools cited in these studies perform health education, prevention and/or health promotion activities, however, it is misleading to refer to themselves as HPS. The main challenges for implementation, development and continuity of HPS were identified as being intersectionality and insufficient financial and qualified human resources. HPS need to be certified and submitted to an ongoing evaluation process. It is also suggested the topic of health promotion be included in the syllabuses of training courses of health education teachers and other health education professionals.
\end{abstract}

Key words Health promotion, Health Promoting Schools, School Health
Resumo O objetivo deste artigo é analisar os critérios de acreditação dos estudos que avaliaram as ações de promoção da saúde e prevenção de fatores de risco das Escolas Promotoras de Saúde (EPSs). Realizou-se uma revisão sistemática conduzida a partir das recomendações propostas no guia Preferred Reporting Items for Systematic Reviewsand Meta-Analyses (PRISMA) dos artigos que avaliaram as EPSs nas bases de dados: SciELO, Lilacs, Medline, PubMed e Portal Capes. A partir da análise dos três eixos para acreditação das EPS, três dos estudos analisados não contemplaram todos os critérios para serem certificadas como EPS nos eixos "Processo de Planejamento" $e$ "Atividades de Promoção de Saúde desenvolvidas". As escolas citadas nestes estudos apresentam atividades de educação em saúde, prevenção e/ ou promoção da saúde, no entanto, é equivocado se autodenominarem EPS. Identificou-se como principais desafios enfrentados para a implantação, o desenvolvimento e a continuidade das EPS, a intersetorialidade e a insuficiência de recursos financeiros e humanos capacitados. As EPSs precisam ser certificadas e submetidas a um processo avaliativo contínuo. Sugere-se ainda a inclusão do tema promoção de saúde nos currículos dos cursos de formação dos professores e outros profissionais da educação e da saúde.

Palavras-chave Promoção da saúde, Escolas promotoras de saúde, Saúde escolar 


\section{Introdução}

A promoção da saúde, processo abrangente e contínuo, que envolve prevenção de fatores de risco, educação e a participação de diferentes setores da sociedade, resgata a concepção da saúde como produção social ${ }^{1,2}$. Este conceito, descrito pela Carta de Ottawa (1986), destaca a criação de ambientes favoráveis para a saúde, pois considera que esta se cria e se vive no marco da vida cotidiana: nos centros de ensino, de trabalho e de lazer. Para isso, diversas estratégias foram adotadas em todo o mundo para a implementação de políticas de promoção da saúde, dentre elas a Escola Promotora de Saúde (EPSs) ${ }^{3}$.

Na década de 1990, a Orgnização Mundial da Saúde (OMS) definiu EPS como aquela que promove, fomenta e permite a aquisição de habilidades pessoais e sociais que buscam criar valores e atitudes positivas sobre a saúde, como a própria capacidade de tomar decisões pessoais, a participação e igualdade 4 . Trata-se de uma estratégia de promoção da saúde no espaço escolar com enfoque integral e trabalho permanente em políticas públicas saudáveis, baseada em três pilares de intervenção na escola e na comunidade: Educação em saúde; Criação, organização e manutenção de ambientes favoráveis à saúde; e, Provisão de serviços de saúde, nutrição saudável e vida ativa com a comunidade, parcerias e serviços escolares ${ }^{5-7}$.

Em 1995, a Organização Pan-Americana da Saúde (OPAS) lançou a Iniciativa Regional de EPSs, desde então, os Estados Membros da América Latina e do Caribe têm fortalecido suas ações a partir de uma rediscussão e reflexão sobre atividades no campo da saúde escolar ${ }^{8}$. As experiências de promoção de saúde em âmbito escolar no Brasil refletem o compromisso em fortalecer a Rede Latino-Americana de EPSs. No Brasil, o trabalho educativo em saúde, vivenciado na escola, além de consolidar a cooperação técnica entre os Ministérios da Saúde e da Educação, tem avançado através da incorporação de novas concepções teóricas da educação e da saúde e, das práticas educativas em saúde no cotidiano didático-pedagógico, assim como na diversificação de seu campo de atuação ${ }^{8}$.

A meta das EPSs é o fortalecimento do desenvolvimento humano sustentável de alunos e demais membros da comunidade escolar, fundamentada em duas bases principais: as declarações e orientações de promoção de saúde; e as considerações de saúde, educação e setores sociais, que considera o contexto e as experiências de outros programas de saúde escolar, os conhecimentos científicos e técnicos sobre saúde e educação, inclusive teorias, modelos e ações educativas ${ }^{5}$.

A criação de EPSs, tem base na ação intersetorial que congrega atores que circulam em diferentes espaços ${ }^{8}$. Suas ações são pautadas no reforço do sujeito social para capacitá-lo a cuidar de si e agir em grupo e em defesa da promoção da saúde; na utilização de estratégias que permitam a coexistência da interface de várias áreas do conhecimento; no reconhecimento da dimensão afetiva no processo de transformação e tomada de decisão, e no incentivo e fomento de parcerias por meio de redes sociais de apoio?.

Com base nas diretrizes gerais da iniciativa EPSs, observa-se avanços no processo de definição de critérios e procedimentos para a acreditação das EPSs. Os procedimentos incluem normas sobre requisitos mínimos, certificação por parte dos Ministérios da Saúde e da Educação, atividades de monitoramento e acompanhamento, requisitos de informação e período de acreditação ${ }^{5}$. O processo de acreditação considera as orientações da iniciativa EPS com a participação de diretores e professores, alunos, pais, administrativos e organizações da comunidade ${ }^{10}$.

Sendo assim, o objetivo dessa revisão sistemática foi analisar os critérios de acreditação dos estudos que avaliaram as ações de promoção da saúde e prevenção de fatores de risco das EPSs.

\section{Métodos}

Trata-se de uma revisão sistemática, conduzido a partir das recomendações propostas no guia Preferred Reporting Items for Systematic Reviewsand Meta-Analyses (PRISMA) ${ }^{11}$, tendo como foco a análise da produção científica que avaliaram ações de promoção da saúde das EPSs.

Realizou-se uma busca bibliográfica dos artigos publicados sobre esta temática nas bases de dados: Scientific Eletronic Library Online (SciELO), Literatura Latino-Americana e do Caribe em Ciências da Saúde (Lilacs), Medical Literature Analysisand Retrieval System Online (Medline), National Library of Medicine, Bethesda, MD (PubMed) e Portal Capes. No intuito de contemplar toda a produção científica o período de publicação não foi delimitado.

Para a busca de dados foram utilizadas as seguintes combinações de descritores e os seus similares em Inglês e Espanhol: 1) Escolas Promotoras de Saúde and Saúde Escolar and Educação em Saúde; 2) Educação em Saúde and Escolas Promotoras de Saúde and Promoção da Saúde e 
3) Educação em Saúde and Escolas Promotoras de Saúde and Prevenção de Doenças.

Os estudos foram selecionados, de maneira independente, por três pesquisadores. Inicialmente, excluíram-se os estudos duplicados. A seguir, selecionaram-se os estudos referentes à temática abordada por meio da leitura dos títulos e resumos. Para a inclusão dos artigos, consideraram-se os estudos originais realizados em escolas que contemplavam os critérios de acreditação de uma EPS, baseado nos critérios adaptados da Organização Pan-Americana de Saúde (Quadro 1). Excluíram-se os estudos de revisão, que utilizavam dados secundários e que não apresentavam metodologia clara e definida.

Extraiu-se dos artigos as seguintes informações: autores, ano de publicação, local de realização, características da amostra, objetivos do estudo e a execução das ações desenvolvidas pelas EPSs segundos os critérios de acreditação, baseado em três eixos principais: processo de planejamento, atividades desenvolvidas e participantes no programa.

\section{Resultado}

A busca resultou na identificação inicial de 621 artigos. Após aplicar os critérios de inclusão, elegeram-se 5 estudos, incluídos na presente revisão (Figura 1).

Os estudos selecionados foram publicados entre 2007 e 2013 e realizados na China, Espanha e no grupo de países da União Europeia. Todos apresentaram como objeto de estudo as EPS (Quadro 2). A avaliação das escolas se deu por meio de entrevistas e/ou questionários aplicados a pelo menos um dos seguintes grupos: alunos, professores, funcionários/administradores das escolas, pais, coordenadores e profissionais da saúde que trabalhavam nos centros educativos.

No eixo Processo de Planejamento, um dos critérios para a acreditação é o documento de compromisso para desenvolvimento das ações das EPSs. Apesar de todos os estudos afirmarem avaliar EPS, em nenhum foi mencionado de forma clara este documento (Quadro 3).

$\mathrm{O}$ estudo de Lee et al. ${ }^{12}$ não mencionou o Plano de Ação e o Grupo de Trabalho para implementação da iniciativa e coordenação com outros setores, que trata da participação de re-

Quadro 1. Critérios para acreditação de uma Escola Promotora de Saúde ${ }^{a}$

\begin{tabular}{|c|c|c|}
\hline $\begin{array}{c}\text { Processo de Planejamento } \\
\text { (A escola deve reunir pelo menos } 3 \\
\text { dos seguintes elementos) }\end{array}$ & $\begin{array}{c}\text { Atividades Desenvolvidas } \\
\text { (A escola deve ter pelo menos } 1 \\
\text { programa, em } 3 \text { das seguintes áreas } \\
\text { prioritárias) }\end{array}$ & $\begin{array}{c}\text { Participantes no Programa } \\
\text { (A escola deve incorporar pelo } \\
\text { menos } 3 \text { participantes dos } \\
\text { seguintes representantes) }\end{array}$ \\
\hline $\begin{array}{l}\text { Documento de Compromisso para } \\
\text { o desenvolvimento da iniciativa }\end{array}$ & $\begin{array}{l}\text { Alimentação saudável (lanchonetes, } \\
\text { cantinas ou refeitórios saudáveis) }\end{array}$ & Pessoal da administração escolar \\
\hline $\begin{array}{l}\text { Grupo de trabalho para } \\
\text { implementação da iniciativa, } \\
\text { que envolva diferentes atores } \\
\text { (administração escolar, professores, } \\
\text { pais, alunos e comunidade) }\end{array}$ & $\begin{array}{l}\text { Educação Física (ampliação das horas } \\
\text { dedicadas à atividade física, recreação } \\
\text { e esportes, melhoramento dos espaços } \\
\text { físicos) }\end{array}$ & Professores \\
\hline $\begin{array}{l}\text { Plano de ação de pelo menos um } \\
\text { ano }\end{array}$ & $\begin{array}{l}\text { Fatores psicossociais protetores } \\
\text { (afetividade e sexualidade, educação } \\
\text { em Habilidades para a vida, relações } \\
\text { interpessoais) }\end{array}$ & Alunos \\
\hline $\begin{array}{l}\text { Inclusão da iniciativa no plano de } \\
\text { ação da comunidade }\end{array}$ & $\begin{array}{l}\text { Uso de tabaco, álcool e outras drogas } \\
\text { (declaração de espaços livres de tabaco, } \\
\text { atividades educativas, prevenção) }\end{array}$ & Pais e mães de família \\
\hline- & $\begin{array}{l}\text { Promoção da saúde bucal (instalação } \\
\text { de espaços adequados para a } \\
\text { escovação, atividades educativas) }\end{array}$ & Representantes da Comunidade \\
\hline- & $\begin{array}{l}\text { Ambiente escolar saudável } \\
\text { (melhoramento dos espaços físicos, } \\
\text { educação ambiental, criação e } \\
\text { conservação de áreas verdes) }\end{array}$ & - \\
\hline
\end{tabular}

${ }^{a}$ Modelo do Chile; Fonte: Adaptado de Organização Pan-Americana de Saúde ${ }^{5}$. 


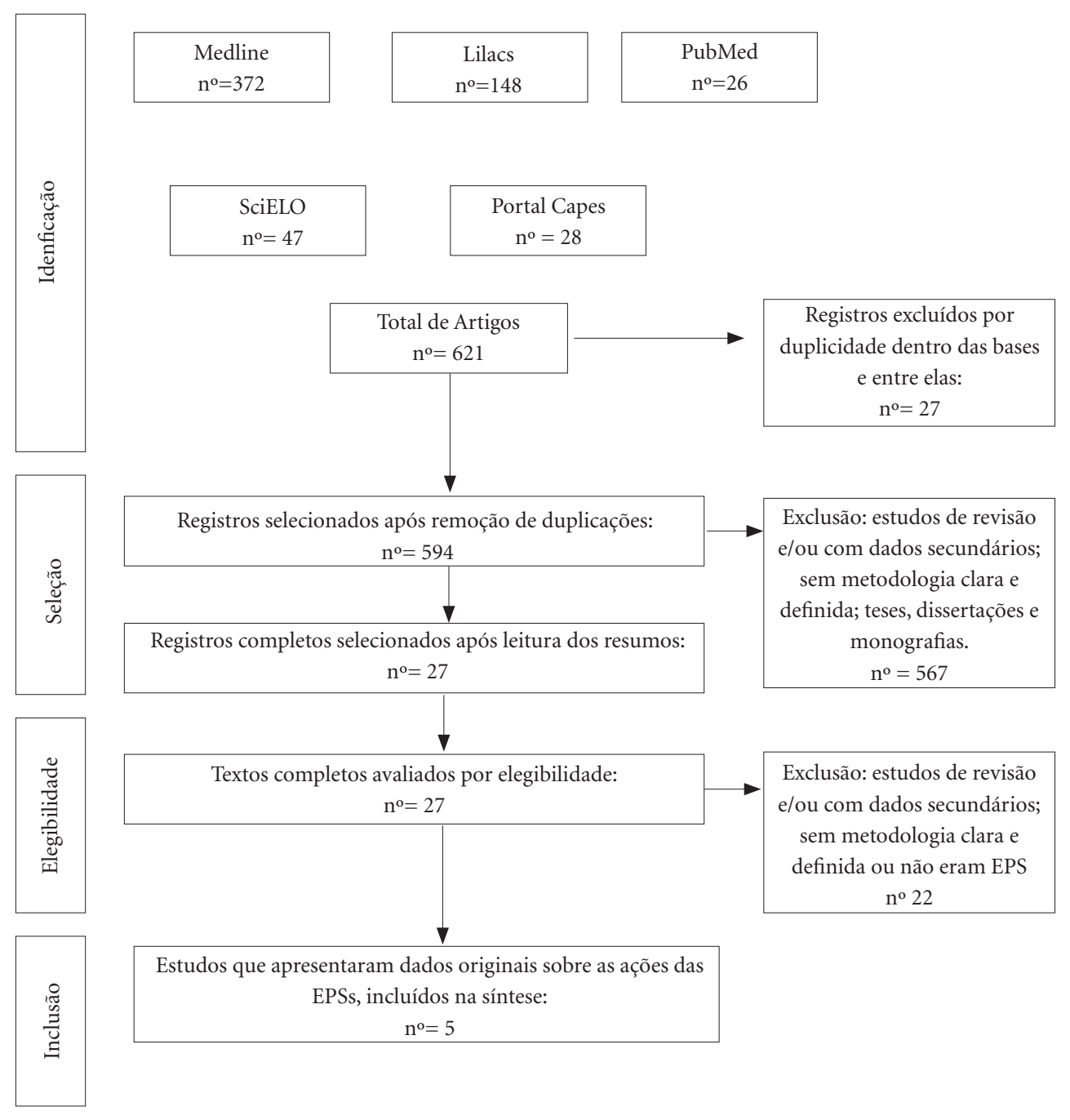

Figura 1. Fluxograma de identificação e seleção dos artigos para revisão sistemática sobre ações das escolas promotoras de saúde em âmbito mundial.

presentantes dos administradores escolares, docentes, pais e mães de família, estudantes e associação de pais e mestres. Apesar de não descrever características da amostra avaliada, sabe-se que professores e diretores responderam ao questionário utilizado pelo Sistema de Acreditação das Escolas Saudáveis e Promotoras de Saúde, desenvolvido pelo Centro de Educação e Promoção da Saúde da Universidade Chinesa de Hong Kong.

De todos os estudos analisados apenas o de Aldinger et al. ${ }^{13}$, não mencionou incluir o Programa de Saúde Escolar no Plano de Ação da Comunidade, no projeto educativo institucional ou nos planos nacionais regulares. Este também foi desenvolvido na China, na província de Zhe- jiang. A fim de compor o Inquérito Quantitativo de Saúde Global de Estudante, foi incentivado pela OMS que desenvolve um projeto na China, com metas específicas e considerações culturais: alcançar uma "educação de qualidade", que não se concentrasse apenas no desempenho acadêmico, mas também no desenvolvimento social, emocional e físico da criança. Especialistas chineses, complementaram a iniciativa da OMS com o desenvolvimento de um instrumento que também avaliasse medidas qualitativas do processo e os procedimentos de execução EPSs e seus participantes.

De forma complementar, ainda referente ao eixo Processo de Planejamento, o estudo de Lee et 
Quadro 2. Informações dos artigos selecionados sobre Escolas Promotoras de Saúde.

\begin{tabular}{|l|l|l|l|}
\hline $\begin{array}{l}\text { Autores e Ano } \\
\text { de Publicação }\end{array}$ & $\begin{array}{l}\text { Local do } \\
\text { Estudo }\end{array}$ & \multicolumn{1}{|c|}{ Amostra do estudo } & \multicolumn{1}{c|}{ Objetivo } \\
\hline $\begin{array}{l}\text { Lee et al., } \\
2007^{12}\end{array}$ & $\begin{array}{l}\text { Hong Kong, } \\
\text { China }\end{array}$ & $\begin{array}{l}\text { Avaliou professores e diretores de 10 } \\
\text { escolas secundárias. }\end{array}$ & $\begin{array}{l}\text { Analisar a implementação do Sistema } \\
\text { de Escolas Saudáveis em Hong Kong. }\end{array}$ \\
\hline $\begin{array}{l}\text { Simovska, } \\
2007^{13}\end{array}$ & $\begin{array}{l}\text { União } \\
\text { Europeia } \\
\text { (UE) }\end{array}$ & $\begin{array}{l}8 \text { classes de } 8 \text { países: } 8 \text { professores, } \\
8 \text { facilitadores e 200 estudantes com } \\
\text { idade entre 13 e 16 anos. }\end{array}$ & $\begin{array}{l}\text { Discutir o envolvimento de alunos } \\
\text { acerca da aprendizagem de questões } \\
\text { de saúde. }\end{array}$ \\
\hline $\begin{array}{l}\text { Aldinger et al., } \\
2008^{14}\end{array}$ & $\begin{array}{l}\text { Província } \\
\text { de Zhejiang, } \\
\text { China }\end{array}$ & $\begin{array}{l}\text { 9 escolas (15.200 alunos). } \\
\text { Entrevistaram: 26 administradores } \\
\text { escolares, 56 professores e funcionários } \\
\text { das escolas, 64 alunos e 45 pais. }\end{array}$ & $\begin{array}{l}\text { Avaliar as mudanças de atitudes, } \\
\text { conhecimentos e comportamentos } \\
\text { após implatação de uma EPS. }\end{array}$ \\
\hline $\begin{array}{l}\text { Simovska et } \\
\text { al., 2012 }\end{array}$ & $\begin{array}{l}27 \text { países } \\
\text { UE }\end{array}$ & $\begin{array}{l}\text { Avaliaram 24 coordenadores da Rede } \\
\text { Europeia de Escolas Saudáveis }\end{array}$ & $\begin{array}{l}\text { Apresentar o projeto HEPS } \\
\text { (Alimentação Saudável e Atividade } \\
\text { Física nas Escolas) discutir as etapas } \\
\text { iniciais da sua implementação. }\end{array}$ \\
\hline $\begin{array}{l}\text { Ramos et al., } \\
2013^{4}\end{array}$ & $\begin{array}{l}\text { Barcelona, } \\
\text { Espanha }\end{array}$ & $\begin{array}{l}12 \text { centros de educação. Entrevistaram: } \\
\text { 24 docentes e 23 profissionais da saúde } \\
\text { que trabalham nos centros educativos. }\end{array}$ & $\begin{array}{l}\text { Avaliar a estratégia Hagamos Salud } \\
\text { nos centros de educação primária e } \\
\text { secundária da cidade de Barcelona em } \\
\text { seu primeiro ano de aplicação. }\end{array}$ \\
\hline
\end{tabular}

Quadro 3. Resultados das ações desenvolvidas pelas Escolas Promotoras de Saúde segundo critérios de acreditação em três eixos proposto pela OPAS (2006). Legenda: X - critério mencionado no estudo; - critério não mencionado no estudo.

\begin{tabular}{|c|c|c|c|c|c|c|}
\hline \multicolumn{7}{|c|}{ Critério: Processo de Planejamento } \\
\hline $\begin{array}{l}\text { Autores e Ano de } \\
\text { Publicação }\end{array}$ & \multicolumn{2}{|c|}{$\begin{array}{c}\text { Documento de } \\
\text { Compromisso para } \\
\text { Desenvolvimento das Ações }\end{array}$} & Grupo de Trabalho & Plano de Ação & \multicolumn{2}{|c|}{$\begin{array}{c}\text { Inclusão da Iniciativa } \\
\text { no Plano de Ação da } \\
\text { Comunidade }\end{array}$} \\
\hline Lee et al., $2007^{12}$ & \multicolumn{2}{|c|}{-} & - & - & \multicolumn{2}{|c|}{$\mathrm{X}$} \\
\hline Simovska, $2007^{13}$ & \multicolumn{2}{|l|}{-} & $\mathrm{X}$ & $\mathrm{X}$ & \multicolumn{2}{|c|}{$\mathrm{X}$} \\
\hline Aldinger et al., $2008^{14}$ & \multicolumn{2}{|l|}{-} & $\mathrm{X}$ & $\mathrm{X}$ & \multicolumn{2}{|c|}{-} \\
\hline Simovska et al., $2012^{15}$ & \multicolumn{2}{|l|}{-} & $\mathrm{X}$ & $\mathrm{X}$ & \multicolumn{2}{|c|}{$\mathrm{X}$} \\
\hline Ramos et al., $2013^{4}$ & \multicolumn{2}{|l|}{-} & $\mathrm{X}$ & $\mathrm{X}$ & \multicolumn{2}{|c|}{$\mathrm{X}$} \\
\hline \multicolumn{7}{|c|}{ Critério: Atividades de Promoção de Saúde Desenvolvidas } \\
\hline $\begin{array}{l}\text { Autores e Ano de } \\
\text { Publicação }\end{array}$ & $\begin{array}{c}\text { Alimentação } \\
\text { Saudável }\end{array}$ & $\begin{array}{l}\text { Educação } \\
\text { Física }\end{array}$ & \begin{tabular}{c|} 
Fatores \\
Psicossociais \\
Protetores \\
\end{tabular} & $\begin{array}{c}\text { Combate ao Uso } \\
\text { de Tabaco, Álcool } \\
\text { e Outras Drogas }\end{array}$ & $\begin{array}{l}\text { Promoção da } \\
\text { Saúde Bucal }\end{array}$ & $\begin{array}{l}\text { Ambiente } \\
\text { Escolar } \\
\text { Saudável }\end{array}$ \\
\hline Lee et al., $2007^{12}$ & $\mathrm{X}$ & - & $\mathrm{X}$ & $\mathrm{X}$ & - & $\mathrm{X}$ \\
\hline Simovska, $2007^{13}$ & $\mathrm{X}$ & - & $\mathrm{X}$ & $\mathrm{X}$ & - & $\mathrm{X}$ \\
\hline Aldinger et al., $2008^{14}$ & $\mathrm{X}$ & $\mathrm{X}$ & $\mathrm{X}$ & $\mathrm{X}$ & - & $\mathrm{X}$ \\
\hline Simovska et al., $2012^{15}$ & $\mathrm{X}$ & $\mathrm{X}$ & - & - & - & $\mathrm{X}$ \\
\hline Ramos et al., $2013^{4}$ & - & - & - & - & - & $\mathrm{X}$ \\
\hline \multicolumn{7}{|c|}{ Critério: Participantes no Programa } \\
\hline $\begin{array}{c}\text { Autores e Ano de } \\
\text { Publicação }\end{array}$ & $\begin{array}{c}\text { Administração } \\
\text { Escolar }\end{array}$ & Professores & Alunos & $\begin{array}{c}\text { Pais e Mães de } \\
\text { Família }\end{array}$ & \multicolumn{2}{|c|}{$\begin{array}{c}\text { Representantes da } \\
\text { Comunidade }\end{array}$} \\
\hline Lee et al., $2007^{12}$ & $\mathrm{X}$ & $\mathrm{X}$ & $\mathrm{X}$ & $\mathrm{X}$ & \multicolumn{2}{|c|}{$\mathrm{X}$} \\
\hline Simovska, $2007^{13}$ & - & $\mathrm{X}$ & $\mathrm{X}$ & - & \multicolumn{2}{|c|}{$\mathrm{X}$} \\
\hline Aldinger et al., $2008^{14}$ & $\mathrm{X}$ & $\mathrm{X}$ & $\mathrm{X}$ & $\mathrm{X}$ & \multicolumn{2}{|c|}{-} \\
\hline Simovska et al., $2012^{15}$ & $\mathrm{X}$ & $\mathrm{X}$ & $\mathrm{X}$ & $\mathrm{X}$ & & $\mathrm{X}$ \\
\hline Ramos et al., $2013^{4}$ & $\mathrm{X}$ & $\mathrm{X}$ & $\mathrm{X}$ & $\mathrm{X}$ & & $\mathrm{X}$ \\
\hline
\end{tabular}


al. ${ }^{12}$ e Aldinger et al. ${ }^{13}$, não contemplaram o mínimo de três aspectos necessários, baseados nas normas para ser acreditado o título de EPS.

No critério de Atividades de Promoção de Saúde desenvolvidas pelas EPS, destacam-se as ações implementadas para avaliar e melhorar a saúde dos alunos, o corpo de professores, membros da família e da comunidade ${ }^{10}$. Todos os estudos analisados apresentam as EPS propiciando um ambiente escolar saudável (Quadro 3), como por exemplo melhoramento dos espaços físicos, condições de saneamento, educação ambiental, criação e conservação de áreas verdes.

Ações voltadas à alimentação saudável (lanchonetes, cantinas ou refeitórios saudáveis) não foram mencionadas pelo estudo de Ramos et al. ${ }^{4}$, o qual mencionou desenvolver apenas a atividade de Ambiente Escolar Saudável.

Já as ações que dizem respeito à educação física (ampliação das horas dedicadas à atividade física, recreação e esportes, melhoramento dos espaços físicos) foram mencionados apenas por dois estudos: Aldinger et al. ${ }^{14}$ desenvolvido na província de Zhejiang (China) incentivado pela OMS, a fim de compor o Inquérito Quantitativo de Saúde Global de Estudante; e pelo estudo de Simovska et al..$^{15}$ que entrevistaram 24 coordenadores em escolas saudáveis do projeto HEPS (Alimentação Saudável e Atividade Física nas Escolas) em 27 países da UE (Quadro 3). Tal projeto, integrante da Rede Escolas Europeias Saudáveis, visa promover saúde e bem-estar e evitar que as crianças se tornem obesas, pois acreditam que o excesso de peso e obesidade estão associados com uma série de consequências para a saúde física e psicossocial.

Outras ações das EPS com enfoque nos fatores psicossociais protetores, como atividades com o tema de afetividade e sexualidade, educação em Habilidades para a Vida e relações interpessoais e enfoque no Combate ao Uso de Tabaco, Álcool e Outras Drogas com atividades que declaram espaços livres de tabaco, atividades educativas e prevenção, foram mencionadas por três estudos: Lee et al. ${ }^{12}$, Aldinger et al..$^{14} \mathrm{e}$ Simovska et al..$^{15}$, que foi baseado no projeto Young Minds, que avaliou 216 voluntários composto por professores, facilitadores e alunos. Tal projeto foi desenvolvido em diferentes países europeus que colaboraram com questões relacionadas à saúde. $\mathrm{O}$ propósito do projeto é gerar conhecimento baseado em pesquisa-ação sobre métodos eficazes para envolver os alunos em aprender sobre saúde em uma ação e colaboração de maneira focada.

Nenhum dos estudos avaliados mencionou desenvolver atividades de Promoção da Saúde Bucal.
A partir das análises dentro do eixo Atividades de Promoção de Saúde desenvolvidas pelas EPS, apenas o estudo de Ramos et al. ${ }^{4}$ não mencionou o mínimo de 3 atividades necessárias para acreditação de EPS.

Por fim, ainda no contexto dos procedimentos e normas sobre requisitos mínimos no processo de acreditação das EPS, o terceiro eixo trata dos Participantes nos programas. As EPSs envolvem todos os membros da escola e da comunidade (incluindo professores, pais, estudantes, líderes e organizações não governamentais) na tomada de decisões e na execução das intervenções para a promoção da aprendizagem, no fomento de estilos de vida saudável e na realização de projetos comunitários de promoção de saúde (5). A partir das análises dos estudos, apenas dois deles não contemplaram todos os representantes envolvidos com a comunidade escolar: Simovska ${ }^{13}$ que não mencionou representantes da administração escolar e pais; e Aldinger et al. ${ }^{14}$ que não mencionou representantes da comunidade. Ainda assim, neste eixo todos os estudos contemplaram o mínimo de 3 critérios (Quadro 3).

Somente dois estudos mencionaram as barreiras, desafios e estratégias para implantação e desenvolvimento das EPS: Simovska et al. ${ }^{15}$ e Ramos et al. ${ }^{4}$. O primeiro avaliou o projeto HEPS (Alimentação Saudável e Atividade Física nas Escolas). Os coordenadores apresentaram consenso em apresentar como principal desafio, recurso financeiro insuficiente. Também relataram tempo insuficiente e falta de recurso humano qualificado. Além da falta de apoio do governo na esfera nacional, regional e inclusive falta de apoio da própria escola também foram mencionadas como desafios. As conclusões apontam que além da falta de apoio e falta de financiamento, também falta clareza nas responsabilidades dos coordenadores nacionais relativas à aplicação do projeto como um todo. Ainda ressaltam que a existência de diversos programas concorrentes, falta de ferramentas na área de promoção da alimentação saudável e da atividade física e desenvolvimento de políticas, implementação e avaliação parecem ser uma barreira para a implementação do conjunto de ferramentas do HEPS.

As principais estratégias para superar estes desafios dizem respeito ao desenvolvimento de políticas para capacitar professores e outros profissionais da educação, além da divulgação das publicações sobre a iniciativa para o Ministério da Educação e Saúde, profissionais, políticos e universidades, responsáveis pelo currículo escolar, assim como apresentar em conferências e se- 
minários. Ressaltam a importância da formação de profissionais de educação, sobretudo professores, e outros profissionais da saúde, baseada no tema da iniciativa do HEPS e sobre a importância da promoção da saúde em meio escolar. Também sugerem incentivar as escolas a avaliar os seus programas.

Já o estudo de Ramos et al. ${ }^{4}$ avaliou as primeiras fases de implantação da estratégia Hagamos Salud! em uma rede de escolas em Barcelona (Espanha). Dentre as barreiras percebidas para implantação da estratégia da EPS, destacam-se a escassez de tempo e recursos humano e econômico disponíveis. Também se detectou a dificuldade de promover a participação e garantir a permanência e a falta de cultura participativa local. Outra barreira apontada foi a delegação das responsabilidades das famílias para os docentes.

De forma complementar, o estudo de Ramos et al. ${ }^{4}$ ressaltou ainda que a participação dos agentes da comunidade educativa foi qualificada unanimemente como ponto forte para garantir a continuidade e o valor da estratégia. Os docentes e profissionais sugeriram incentivar os centros educativos com o certificado de acreditação de EPS, proporcionando reconhecimento, além de permitir que cada centro tenha seu próprio ritmo e controle da difusão dos primeiros anos de implantação para assegurar a qualidade do projeto. E como consenso, a principal estratégia sugerida para vencer esses desafios foi a formação continuada e permanente dos professores, assim como espaços de encontros entre os agentes envolvidos que permite compartilhar experiências, proporcionar motivação e trocar ideias.

\section{Discussão}

A partir da análise dos três eixos para acreditação das EPSs (Processo de Planejamento, Atividades Desenvolvidas e Participantes no Programa), três dos cinco estudos não mencionaram informações consistentes ${ }^{4,12,14}$. Embora as escolas citadas nestes estudos apresentassem atividades de educação em saúde, prevenção de doenças e acidentes e/ou promoção da saúde, não contemplaram todos os critérios para serem certificadas como EPS.

Uma revisão sobre saúde escolar na América Latina ${ }^{16}$ apontou que a escola tem representado um importante local para o encontro entre saúde e educação abrigando amplas possibilidades de iniciativas, como: ações de diagnóstico clínico e/ ou social, estratégias de triagem e/ou encaminhamento aos serviços de saúde especializados ou de atenção básica; atividades de educação em saúde e promoção da saúde. Estas iniciativas têm sido identificadas sob o termo Saúde Escolar utilizado para designar ações que objetivam proporcionar condições adequadas à realização do processo educacional que requer condições mínimas de saúde ${ }^{16}$.

Destaca-se que dois estudos ${ }^{12,14}$ não atenderam aos critérios referentes ao Processo de Planejamento, comprometendo o compromisso dessas escolas para o desenvolvimento da iniciativa EPS e suas diretrizes. Estes se referem a programas de escolas saudáveis classificados dentro da estratégia EPS, o que sugere que essas escolas possuem esses documentos, no entanto não os mencionaram. Outro estudo ${ }^{4}$ não apresentou os critérios de acreditação relacionados ao eixo Atividades de Promoção de Saúde desenvolvidas pelas EPS, descaracterizando a EPS, uma vez que essas ações refletem de fato a efetividade da iniciativa.

Para evitar que escolas com atividades voltadas para a promoção da saúde, sem atender a todos os critérios de acreditação, se autodenominem EPS equivocadamente, a proposta do presente estudo é que a Organização Mundial da Saúde e seus representantes regionais façam uma supervisão mais criteriosa e contínua, baseada na renovação do credenciamento por meio de avaliação periódica. No estudo de Ramos et al. ${ }^{4}$, foi discutido como ponto relevante e uma forma de incentivar os centros educativos, o reconhecimento com a entrega de um certificado de acreditação de EPS para as escolas que atendessem os critérios. Já o estudo de Simovska et al. ${ }^{14}$, apontou a falta do desenvolvimento de políticas de implementação e avaliação, como barreira para a implementação de ações nas EPS e incentivaram a avaliação do programa também pelas escolas.

Em relação aos participantes das escolas promotoras de saúde, apenas dois estudos não mencionaram a participação de todos os representantes envolvidos com a comunidade escolar ${ }^{15,13}$, ainda assim, todos apresentaram o mínimo de 3 critérios para acreditação. Corroborando com esses resultados, em uma revisão ${ }^{16}$ que reflete sobre a Promoção de Saúde na Escola na América Latina, foi observado que há uma preocupação em enfatizar a participação ampla (profissionais, pais, estudantes e comunidades) nas diversas etapas dos projetos e ações.

A participação dos agentes da comunidade educativa foi qualificada como ponto primordial para garantir a continuidade e o valor da estratégia, em um estudo que avaliou professores e profissionais da saúde que atua em EPSs ${ }^{4}$. 
No entanto, este estudo aponta como barreira à implantação das EPSs, a delegação das responsabilidades das famílias para os docentes. Em outro estudo ${ }^{15}$, foi relatado a falta de clareza quanto as responsabilidades dos coordenadores nacionais relativas à aplicação do projeto como um todo, como um desafio enfrentado. Assim, a participação e o cumprimento das funções e responsabilidades de todos os envolvidos é essencial para que a estratégia EPS tenha efetividade.

Nesta abordagem, a escola torna-se um centro de convergência em que professores, estudantes, famílias e comunidade trabalham para um objetivo comum: promover nas crianças o desenvolvimento de habilidades para a vida, que as formem como agentes do desenvolvimento, com autoestima, valores criativos, confiantes e inovadores, críticos, cívicos e morais que levam à busca constante do bem-estar individual e coletivo para a promoção do desenvolvimento humano sustentável ${ }^{17}$.

Há, no entanto, um debate ainda em construção que se refere à definição de papéis e responsabilidades dos envolvidos no planejamento e execução de programas de saúde escolar. Este desafio remete a três conceitos que podem e devem ser aprimorados e problematizados na perspectiva de atuação em saúde escolar, são eles: intersetorialidade, interdisciplinaridade e participação. Esta constatação não é uma novidade em termos de políticas de promoção da saúde ${ }^{16}$. Em uma revisão sobre saúde escolar na América Latina, a intersetorialidade foi considerada como uma das principais barreiras para a efetivação da EPS na Região, sendo recomendado investir na consolidação de mecanismos formais de coordenação intersetorial ${ }^{16}$.

Assim, as EPSs colocam em andamento estratégias que fomentam e apoiam a aprendizagem e a saúde, utilizando-se de todos os meios e recursos disponíveis, envolvendo o setor saúde e o educação e líderes comunitários no desenvolvimento de atividades escolares planificadas. Além do mais, implementam ações para avaliar e melhorar a saúde dos estudantes, da comunidade educativa, das famílias e dos membros da comunidade em geral. Para isso, trabalha com os líderes comunitários para garantir o acesso à nutrição, à atividade física, à administração, aos serviços de saúde e de referência. Inclusive, participam ativamente as associações de pais, as organizações não governamentais e outras organizações na comunidade ${ }^{10}$.

Outro componente fundamental na promoção de saúde no âmbito escolar, diz respeito as
EPSs promovendo um Ambiente Escolar Saudável $^{16}$. Nesta revisão, isso foi confirmado, pois todos os estudos mencionaram promover Ambiente Escolar Saudável. O espaço escolar permite a oportunidade de voltar-se para as crianças como agentes de mudança. Ao compreender a aplicação de ferramentas metodológicas, pode contribuir para mudanças positivas e sustentáveis em conhecimentos, atitudes e comportamentos relacionados com o cuidado do meio ambiente e, assim, melhorar e proteger o ambiente em que operam comportamentos ${ }^{17}$.

No entanto, promover um Ambiente Escolar Saudável, aparece como um dos principais desafios para ser aplicado nas escolas. Em um estudo ${ }^{18}$ que avaliou 238 professores, o domínio "ambiente escolar" foi o que apresentou menor grau de satisfação e mostrou-se o mais prejudicado, englobando os aspectos referentes à falta, insuficiência, inadequação ou insatisfação relacionados ao dinheiro, informações, ambiente de trabalho e serviços de saúde. De forma semelhante, Ramos et al. ${ }^{4}$ e Simovska et al. ${ }^{15}$ apontam que o principal desafio enfrentado para implantação das estratégia das EPS e promover um ambiente escolar saudável, também diz respeito a escassez de tempo e recursos econômicos disponíveis.

Promover um Ambiente Escolar Saudável, pode ser um tanto quanto complexo. Para compreender melhor a complexidade deste tema, a OPAS $^{5}$ esclarece que, a criação de entornos saudáveis devem intercalar duas dimensões diferentes e complementares: a Dimensão física que refere-se ao entorno físico onde se ensina e se aprende, o qual deve garantir as condições mínimas de segurança e saneamento básico que favoreçam a saúde, o bem-estar e o desenvolvimento do potencial dos alunos e demais membros da comunidade educativa; e a Dimensão psicossocial que procura promover a adaptação de um clima de interação harmônica, agradável, respeitadora dos direitos humanos, equitativa, e livre de qualquer forma de violência entre os seus membros, através do ensino da tolerância, democracia e solidariedade.

No estudo de Valdez et al. ${ }^{17}$, são apontadas as dificuldades para promover a educação e cultura para a paz nas escolas, uma vez que existe a violência estrutural de sistemas políticos, sociais e econômicos em diferentes países, o que provoca desigualdades sociais e pobreza. A OPAS, em 1994, por intermédio da Declaração da Conferência Interamericana sobre Sociedade, Violência e Saúde, assume a violência como um problema de saúde ${ }^{19}$.

A segurança no espaço escolar, no que tange ao ambiente físico, emocional e psicológico, é ob- 
jeto de constante preocupação de responsáveis, professores e direção da escola ${ }^{19}$.

A discussão sobre um ambiente escolar seguro e o aspecto da violência, está relacionado a outro critério para a acreditação de uma EPS que são as ações desenvolvidas dentro da dimensão Fatores Psicossociais Protetores, mencionados no presente estudo por Lee et al. ${ }^{12}$, Simovska ${ }^{13}$ e Aldinger et al. ${ }^{14}$.

Nesse sentido é recomendado desenvolver normas sociais que desaprovem o bullying e todas as formas de violência; e estabelecer um clima de relacionamento que demonstre respeito, apoio e cuidado, e que não tolere o bullying ${ }^{19}$. Sendo este último um problema grave, frequente e atual ${ }^{20}$, principalmente no ambiente escolar. Pesquisadores de todo o mundo atentam para esse fenômeno e apontam aspectos preocupantes quanto ao seu crescimento e ao fato de atingir inclusive os primeiros anos de escolarização. Calcula-se que em torno de 5\% a 35\% das crianças em idade escolar estejam envolvidas de alguma forma em condutas agressivas na escola, atuando como vítimas ou agressoras ${ }^{21}$.

A Promoção de Saúde Bucal foi a única atividade que não foi mencionada por nenhum dos estudos desta revisão como atividade desenvolvida na escola. Evidenciando a gravidade do problema, um estudo desenvolvido no município de Goiânia (GO) avaliou 1947 escolares (rede pública e privada) e encontrou $64 \%$ de prevalência de cárie dentária. A fluorose esteve presente em $5,6 \%$ da amostra, sendo mais frequente em escolares da rede privada do que da rede pública ${ }^{22}$. Estes resultados alarmantes, fazem um convite à reflexão da real importância da Promoção de Saúde Bucal nas escolas, negligenciadas ou omitidas pelos estudos desta revisão.

Visto isso, é importante ressaltar que as atividades desenvolvidas pelas EPSs, não se limitam apenas as atividades que compõe os critérios de acreditação. Estas representam ações fundamentais e atendem as necessidades mais prioritárias em uma dimensão global. Cada EPS deverá planejar as atividades baseadas na realidade local. Para garantir que a implantação da estratégia EPS tenha continuidade e efetividade, foi sugerido por um grupo de professores e profissionais da saúde, que cada centro educativo tenha seu próprio ritmo, controle a difusão das suas ações e assim assegurem a qualidade do projeto ${ }^{4}$. No entanto, ficar limitado aos critérios de acreditação das EPSs numa dimensão estritamente objetiva, poderia representar uma desvalorização aos avanços obtidos pelas políticas de promoção de saúde no ambiente escolar. Dessa forma, todas as ações nesse contexto devem ser reconhecidas e valorizadas, mesmo que as escolas que as desenvolvam não sejam acreditadas como EPSs.

Neste sentido, a $\mathrm{OPAS}^{5}$ complementa que uma EPS, orienta a comunidade educativa para que os escolares tenham acesso a todas as ações de prevenção (imunizações, avaliação visual e auditiva, saúde bucal, saúde mental e administração, entre outros) e tratamento de doenças, alimentação e nutrição aos quais tem direito dentro dos mecanismos previstos no sistema sanitário vigente. Além disso, deve informar e educar a comunidade escolar sobre os direitos e deveres dos escolares e dos demais membros dentro do sistema sanitário ao qual pertencem e participam na vigilância epidemiológica da condições de risco e fatores protetores para a saúde aos quais está exposta a comunidade educativa.

É mais fácil promover a aquisição de hábitos e estilos de vida mais saudáveis durante a infância do que mudar maus hábitos consolidados e estruturados na vida adulta. Assim, o trabalho de educação em saúde na infância e adolescência desempenha um papel importante na prevenção de doenças e promoção da saúde, que deve estar associada a ações específicas com as famílias e ao nível da comunidade ${ }^{17}$.

De forma bem coerente e que vem ao encontro das propostas da OPAS 5 , para o sucesso da implantação da EPS, Ramos et al. ${ }^{4}$ apresentou como resultado entre todos os professores e profissionais da saúde entrevistados, que a principal estratégia para implantar as EPSs com êxito, é a formação continuada e permanente dos professores, assim como a criação de um espaço de encontro entre os agentes envolvidos. Além disso, outro estudo ${ }^{15}$ que entrevistou 24 coordenadores da Rede Europeia de Escolas Saudáveis, apresenta como resultado que o principal plano estratégico para o êxito da implantação e continuidade da iniciativa seria o desenvolvimento de treinamento e formação de professores e outros profissionais da educação e da saúde com ênfase na promoção da saúde no ambiente escolar. Sugere também a divulgação das publicações sobre a iniciativa (diretrizes, conceitos, objetivos, dados de efetividade da EPSs) para Ministério da Educação e da Saúde, Universidades, profissionais da educação e saúde que já estão atuando, responsáveis pelo currículo escolar e políticos. Assim como apresentar essas informações em conferências e seminários.

Diante dos principais desafios e barreiras enfrentados para implantação, desenvolvimento 
e continuidade das EPS, outra estratégia sugerida pelo presente estudo, é acrescentar nos currículos dos cursos de formação de professores e outros profissionais da educação e saúde, o tema Promoção da Saúde, o Desenvolvimento de Entornos Saudáveis e EPSs. Nesta mesma perspectiva a OPAS $^{5}$ sugere a inclusão do componente de promoção da saúde no âmbito escolar nos planos curriculares das instituições formadoras de recursos humanos em saúde e educação nos Estados Membros.

As atividades em saúde no espaço escolar devem favorecer uma ação mais reflexiva e crítica do conceito de saúde, com investigação de demandas e temas pertinentes à comunidade escolar e particularmente aos escolares ${ }^{23}$. Assim, a construção de estratégias de promoção da saúde na escola coexiste com ações construídas a partir dos ideais de outros paradigmas ${ }^{16}$.

Quanto às limitações desta revisão, destacam-se a dificuldade em encontrar documentos com os critérios de acreditação e diretrizes das EPSs e estudos com metodologia bem definida de avaliação das ações das EPSs credenciadas e/ou que utilizassem os devidos descritores. Por outro lado, até o momento é desconhecido dos autores dessa revisão, outro estudo que analisou os critérios de acreditação das EPSs e seus desafios e estratégias para implantação e continuidade da iniciativa, sendo este o primeiro. Dada a importância dessas informações para o planejamento, avaliação e reorientação da iniciativa, sugere-se que mais evidências sobre todo o processo de acreditação sejam publicadas pelas EPSs, inclusi- ve no Brasil, em que nenhum estudo foi identificado por essa revisão.

\section{Considerações finais}

Os achados desta revisão sistemática, apontaram para o fato de que grande parte dos estudos se autodenominam como EPS, apesar de não contemplarem todos os critérios para serem certificadas como tais. A acreditação das EPS é baseada em critérios e deveriam ser certificadas pela OMS e/ou pelos seus representantes regionais quando contemplarem as exigências mínimas. Além do mais, o processo de avaliação das EPSs deveria se contínuo e sistemático para renovação do certificado.

A intersetorialidade apresenta ser o principal desafio para a implantação e continuidade das EPSs, assim como a definição e cumprimento das funções de cada membro participante da comunidade escolar, seguido da dificuldade em proporcionar um ambiente saudável, principalmente pela escassez de recursos financeiros e humanos capacitados.

A principal estratégia para a efetividade da implantação da EPS foi considerada o processo de formação dos professores e outros profissionais da educação e da saúde, e sugere incluir nos currículos dos cursos, o tema promoção de saúde, criação de entornos saudáveis e EPSs. Além disso, destaca-se a importância da divulgação de documentos da iniciativa EPS em instituições de ensino, órgãos públicos e em eventos que atinjam a população geral, como conferências e seminários. 


\section{Colaboradores}

MRI Silva e AP Almeida: concepção e desenho da pesquisa; obtenção de dados; análise e interpretação dos dados; redação do manuscrito; revisão crítica do manuscrito quanto ao conteúdo intelectual importante. JC Machado e LS Silva: revisão crítica do manuscrito quanto ao conteúdo intelectual importante. JAF Cardoso: obtenção de dados e redação do manuscrito. GD Costa e RMM Cotta: concepção e desenho da pesquisa e revisão crítica do manuscrito quanto ao conteúdo intelectual importante.

\section{Referências}

1. Sícoli JL, Nascimento PR. Promoção de saúde: concepções, princípios e Operacionalização. Comunic, Saúde, Educ 2003; 7(12):101-122.

2. Souza EM, Grundy E. Promoção da saúde, epidemiologia social e capital social: inter-relações e perspectivas para a saúde pública. Cad Saúde Pública 2004; 20(5):1354-1360.

3. Brasil. Ministério da Saúde (MS). Projeto Promoção da Saúde. As Cartas da Promoção da Saúde. Brasilia: MS; 2002.

4. Ramos P, Pasarín MI, Artazcoz L, Díez E, Juárez O, González I. Escuelas saludables y participativas: evaluación de una estrategia de salud pública. Gac Sanit 2013; 27(2):104-110.

5. Organização Pan-Americana da Saúde (OPAS). Escolas Promotoras de Saúde - Fortalecimento da Iniciativa Regional. Estratégias e linhas de ação 2003-2012. Pan Am Health Organ 2006; 4:72.

6. Moreira GF. Redução de danos do uso de drogas no contexto da escola promotora de saúde. Cien Saude Colet 2006; 11(3):807-816.

7. Figueiredo TAM, Machado VLT, Abreu MMS. A saúde na escola: um breve resgate histórico. Cien Saude Colet 2010; 15(2):397-402.

8. Brasil. Ministério da Saúde (MS), Organização Pan-Americana da Saúde (OPAS). Escolas Promotoras de Saúde: experiências no Brasil. Brasília: MS; 2007.

9. Catrib AMF, Pordeus AMJ, Ataíde MBC, Albuquerque VLM, Vieira NFC. Saúde no Espaço Escolar. In: Barroso MGT, Vieira NFC, Varela ZMV, organizadores. Educação em saúde no contexto da promoção humana. Fortaleza: Edições Demócrito Rocha; 2003. p. 39-46.

10. Ippolito-Shepherd J, Cerqueira YMT. Las escuelas promotoras de la salud en las Américas: una iniciativa regional. México: FNA; 2003.

11. Moher DLA, Tetzlaff J, Altman DG, Prisma Group. Preferred reporting items for systematic reviews and meta-analyses: the PRISMA statement. Ann Intern Med 2009; 151(4):264-269.

12. Lee A, FFK Cheng, H Yuen, M Ho, A Lo, Y Fung, Leung T. Achieving good standards in health promoting schools: Preliminary analysis one year after the implementation of the Hong Kong Healthy Schools Award scheme. Public Health 2007; 121(10):752-760.

13. Simovska V. The changing meanings of participation in school-based health education and health promotion: the participants' voices. Health Educ Res 2007; 22(6):864-878.

14. Aldinger C, Zhang XW, Liu LQ, Pan XD, Yu SH, Jones J, Kass J. Changes in attitudes, knowledge and behavior associated with implementing a comprehensive school health program in a province of China. Health Educ Res 2008; 23(3):1049-1067.

15. Simovska V, Dadaczynski K, Woynarowska B. Healthy eating and physical activity in schools in Europe A toolkit for policy development and its implementation. Health Education 2012; 112(6):513-524.

16. Casemiro JP, Fonseca ABC, Secco FVM. Promover saúde na escola: reflexões a partir de uma revisão sobre saúde escolar na América Latina. Cien Saude Colet 2014; 19(3):829-840. 
17. Valdez EA, Loreto CR, Fernández PB, Pérez HH, Bahena SC, Monreal LA. Promoción de hábitos saludables en escolares de Hermosillo, Sonora, México. Rev Cubana Hig Epidemiol 2012; 50(3):354-364.

18. Penteado RZ, Pereira IMTB. Qualidade de vida e saúde vocal de professors. Rev Saude Publica 2007; 41(2):236243.

19. Liberal EF, Aires RT, Aires MT, Osório ACA. Escola segura. Jornal de Pediatria 2005; 81(Supl. 5):155-163.

20. Trevisol MT, Dresch D. Escola e bullying: a compreensão dos educadores. Revista Múltiplas Leituras 2011; $4(2): 1-2$.

21. Souza CP, Almeida LCP. Bullying em Ambiente Escolar. Goiânia: Enciclopédia Biosfera; 2011. Vol. 7.

22. Freire MCM, Reis SCGB, Gonçalves MM, Balbo PL, Leles CR. Condição de saúde bucal em escolares de 12 anos de escolas públicas e privadas de Goiânia, Brasil. Rev Panam Salud Publica 2010; 28(2):86-91.

23. Cardoso V, Reis AP, Iervolino SA. Escolas promotoras de saúde. Revista Brasileira de Crescimento e Desenvolvimento 2008 ; 18(2):107-115.

Artigo apresentado em 28/08/2016

Aprovado em 16/03/2017

Versão final apresentada em 18/03/2017 